\title{
Functionalizing cellulose nanocrystal with Click modifiable carbohydrate-binding modules
}

Kevin Aïssa, Muzaffer A. Karaaslan, Scott Renneckar and Jack N. Saddler*

\section{Supporting Information.}

Supporting figure 1. SDS-Page picture of carbohydrate-binding modules conjugation ...............2 Supporting figure 2. AFM images of CNCs CBM-PEG grafted, where the adhesion maps of the adhesion profiles is superposed to the 3D height map for the control (a) and modified (b). . .3 Supporting figure 3: AFM images of CNCs CBM-PEG grafted, height only, of the control (a) and modified (b). Apparent orientation of the CNC network in high concentration during sample preparation. Such pattern is occurring sporadically in the CBM-PEG grafted CNC. It only occurs in high concentration of $\mathrm{CNC}$, typically over $0.5 \% \mathrm{wt}$. 


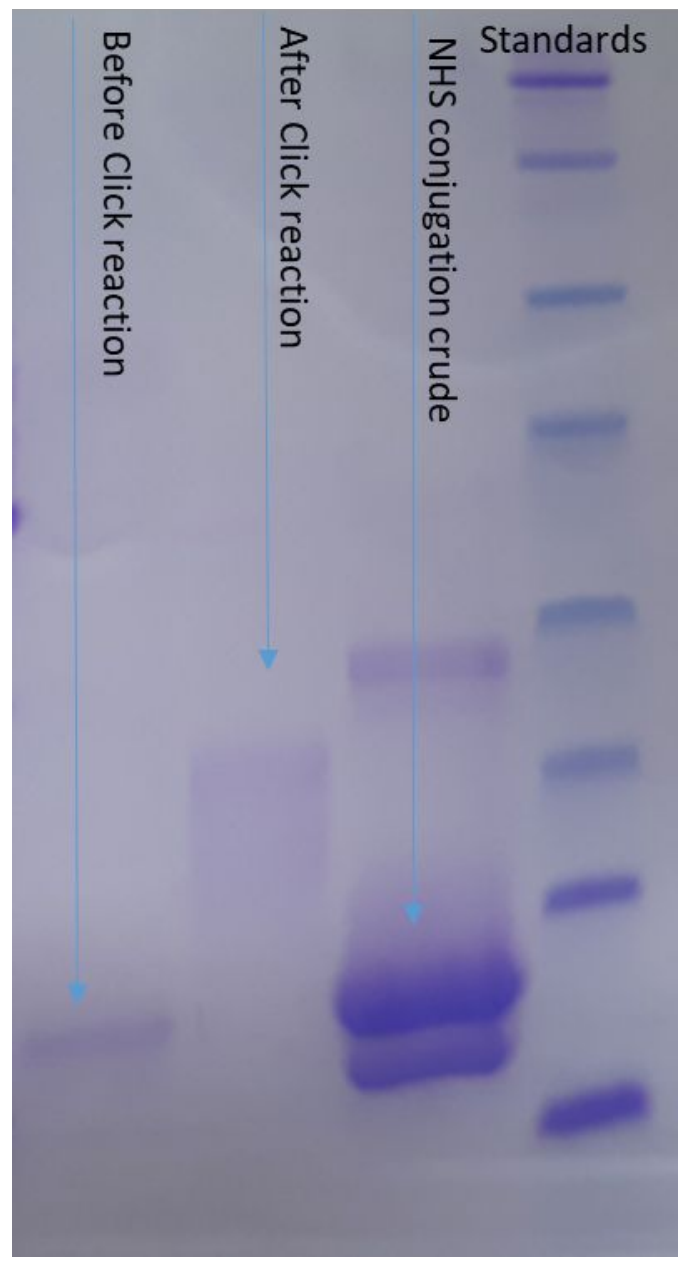

Supporting figure 1. SDS-Page picture of carbohydrate-binding modules conjugation. The polyacrylamide gel was performed on precast gel (4-12\% Criterion ${ }^{\mathrm{TM}}$ XT Bis-Tris Protein Gel, 18 well, $30 \mu$, Bio Rad, Hercules, CA, USA) using XT-MES buffer, XT reducing agent (Bio Rad) The gel was run at 200V, 2A for 40min. Proteins where revealed using brilliant comassie blue (Bio Rad) and destaining solution. 

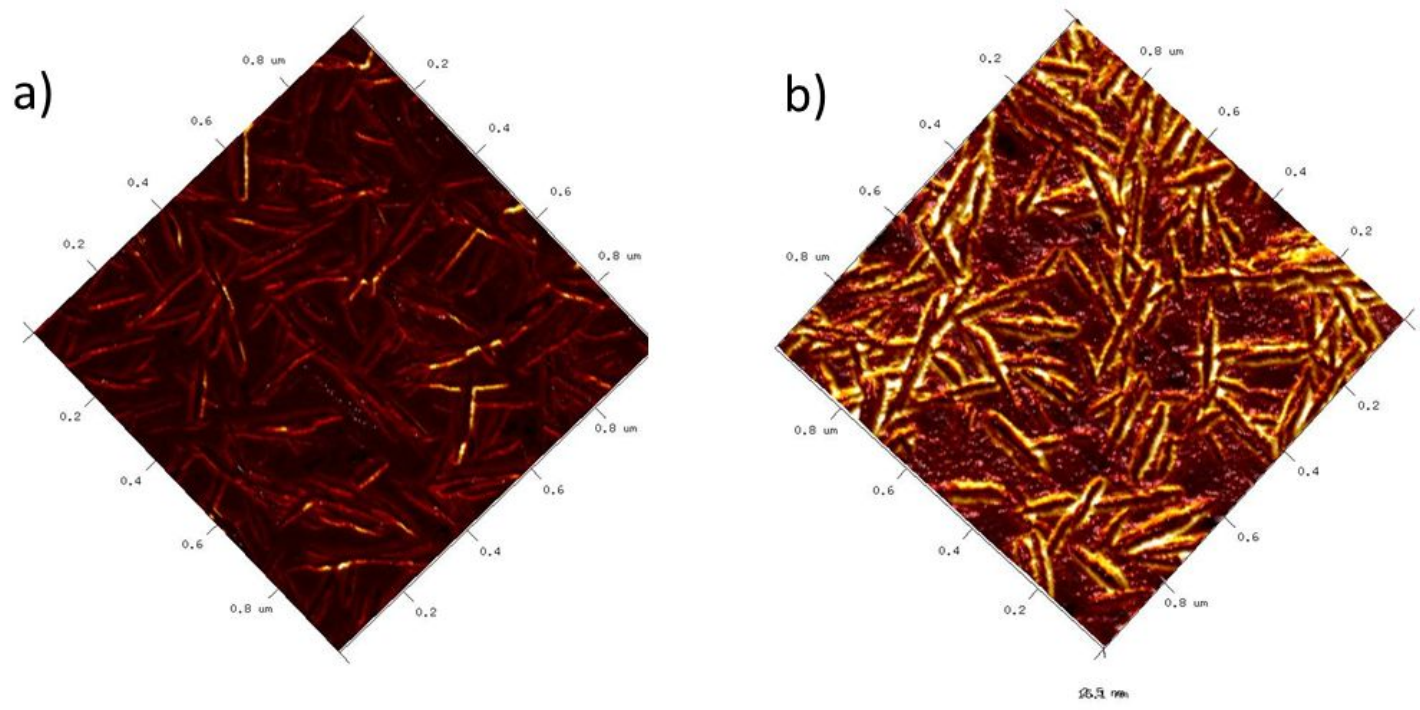

Supporting figure 2. AFM images of CNCs CBM-PEG grafted, where the adhesion maps of the adhesion profiles is superposed to the 3D height map for the control (a) and modified (b).

a)

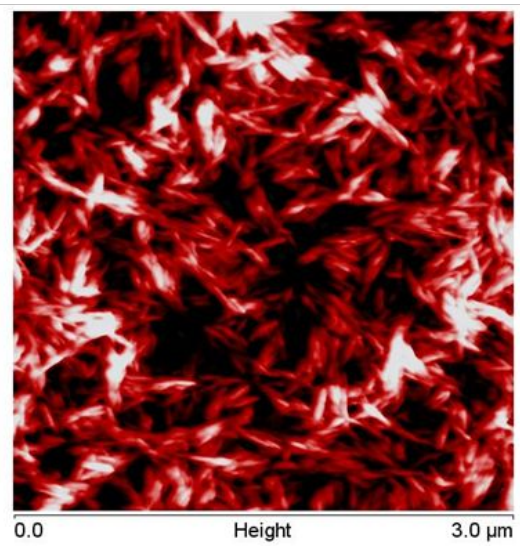

b)

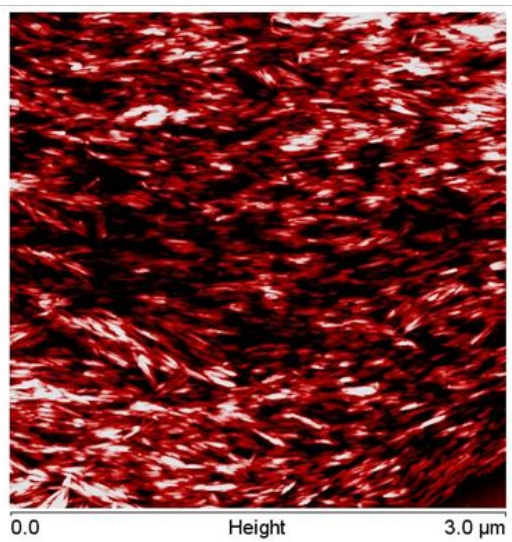

$16.6 \mathrm{~nm}$
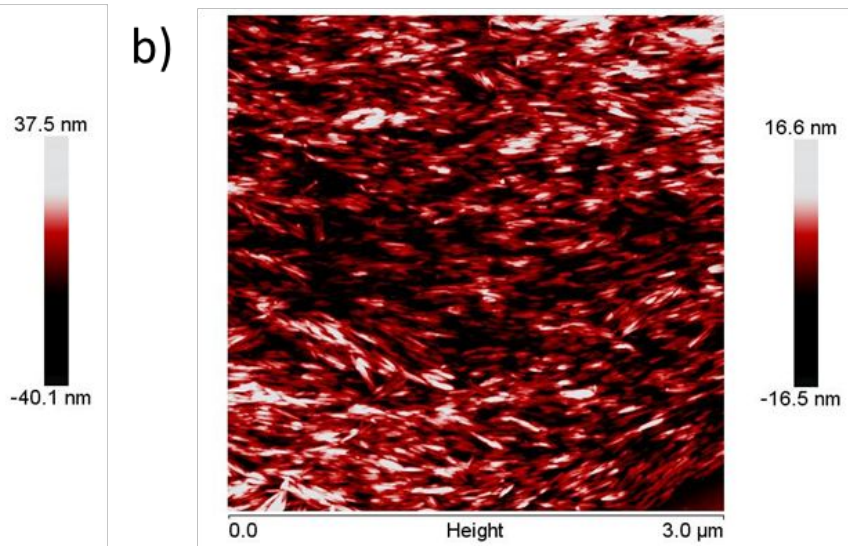

Supporting figure 3: AFM images of CNCs CBM-PEG grafted, height only, of the control (a) and modified (b). Apparent orientation of the $\mathrm{CNC}$ network in high concentration during sample preparation. Such pattern is occurring sporadically in the CBM-PEG grafted CNC. It only occurs in high concentration of $\mathrm{CNC}$, typically over $0.5 \% \mathrm{wt}$. 